\title{
A quantum algorithm for obtaining the energy spectrum of a physical system without guessing its eigenstates
}

\author{
Hefeng Wang* \\ Department of Applied Physics, Xi'an Jiaotong University, Xi'an 710049, China
}

\begin{abstract}
We present a quantum algorithm that provides a general approach for obtaining the energy spectrum of a physical system without making a guess on its eigenstates. In this algorithm, a probe qubit is coupled to a quantum register $R$ which consists of one ancilla qubit and a $n$-qubit register that represents the system. $R$ is prepared in a general reference state, and a general excitation operator acts on $R$ is constructed. The probe exhibits a dynamical response only when it is resonant with a transition from the reference state to an excited state of $R$ which contains the eigenstates of the system. By varying the probe's frequency, the energy spectrum and the eigenstates of the system can be obtained.
\end{abstract}

PACS numbers: 03.67.Ac, 03.67.Lx

\footnotetext{
* Correspondence to wanghf@mail.xjtu.edu.cn
} 


\section{INTRODUCTION}

A fundamental problem in the field of many-body systems is to find efficient ways of simulating Schrödinger equations. The main difficulty is that the dimension of the Hilbert space describing a system of $n$-particles scales exponentially with $n$. This makes direct numerical simulation of a large system intractable. On a quantum computer, however, the number of qubits required to simulate the system increases linearly with the size of the system. And such problems can be solved efficiently on a quantum computer.

In quantum chemistry and computational physics, one often has to diagonalize a large Hamiltonian matrix to obtain the desired eigenvectors and eigenvalues of a system. Classically, the Davidson's algorithm [1] is a large-scale, iterative method which is particularly effective for extracting selected eigenvectors of a Hamiltonian matrix. In this algorithm, one has to use a trial wave function. For large systems, however, this method is expensive and may suffer from slow convergence. Usually this is due to the fact that the trial wave function is not a good approximation to the eigenvector of the Hamiltonian matrix. And it is difficult to find a good trial wave function for a large complicated system, especially when describing excited states.

In quantum computation, the phase estimation algorithm (PEA) can be used for obtaining the eigenvalues of a system [2]. In this algorithm, one guesses an approximated eigenstate of the system, and prepares this guess state as input for the algorithm on a quantum computer. The success probability of the PEA depends on the overlap of the guess state with the real eigenstate of the system. However, in some cases such as the bond-dissociation process in chemistry or that related to excited states of a system, it can become impossible to have a guess state that has any substantial overlap with the desired eigenstates [3]. For many complicated systems, it is very difficult to make even qualitatively correct guess on their eigenstates, or to prepare such states on a quantum computer efficiently.

In Ref. [4], we proposed another quantum algorithm for obtaining the energy spectrum of a physical system. In this algorithm, one also has to make guess on the energy eigenstates of the system. The guess state does not need to have large overlap with any particular eigenstate, but it must have large overlap with one of the eigenstates of the system in order to achieve high efficiency. 


\section{THE ALGORITHM}

In this paper, we present a quantum algorithm for obtaining the energy spectrum of a physical system that we have no knowledge about its eigenstates. This algorithm has the following advantages: $(i)$ one does not need to make a guess on any energy eigenstates of the system; (ii) several adjustable elements (evolution time and system-probe coupling strength) can be varied to improve the efficiency and accuracy of the algorithm; (iii) by introducing a general reference state and a general excitation operator that can be applied for any arbitrary physical system, this algorithm provides a general approach for obtaining the energy spectrum and eigenstates of a system. The details of the algorithm are shown below.

We let a probe qubit couple to a $(n+1)$-qubit quantum register $R$, which contains one ancilla qubit and a $n$-qubit quantum register that represents a physical system of dimension $N=2^{n}$. The Hamiltonian of the whole system is constructed in the form

$$
H=\frac{1}{2} \omega \sigma_{z} \otimes I_{2}^{\otimes(n+1)}+I_{2} \otimes \widetilde{H}+c \sigma_{x} \otimes A,
$$

where $I_{2}$ is the two-dimensional identity operator. The first term in the above equation is the Hamiltonian of the probe qubit, the second term is the Hamiltonian of the register $R$, and the third term describes the interaction between the probe qubit and $R$. Here, $\omega$ is the frequency of the probe qubit $(\hbar=1)$, and $c$ is the coupling strength between the probe qubit and $R$, whereas $\sigma_{x}$ and $\sigma_{z}$ are the Pauli matrices. The Hamiltonian of the register $R$ is in the form

$$
\widetilde{H}=\alpha|0\rangle\left\langle 0\left|\otimes I_{N}+\right| 1\right\rangle\langle 1| \otimes H_{S}
$$

where $I_{N}$ is the $N$-dimensional identity operator; $\alpha$ is a parameter that is set as a reference point for the eigenenergy of the system; $H_{S}$ is the Hamiltonian of the system with dimension of $N$. The operator $A$ is defined as:

$$
A=\sigma_{x} \otimes\left[\frac{1}{\sqrt{2}}\left(I_{2}+\sigma_{x}\right)\right]^{\otimes n} .
$$

It acts on the state space of $R$ and plays the role of an excitation operator.

To run the algorithm, first we prepare the probe qubit in its excited state $|1\rangle$ and the register $R$ in a reference state

$$
\left|\Psi_{0}\right\rangle=\frac{1}{\sqrt{N}} \sum_{j=1}^{N}\left|\varphi_{j}\right\rangle=|0\rangle \otimes\left(\frac{1}{\sqrt{N}} \sum_{j=1}^{N}|j-1\rangle\right),
$$




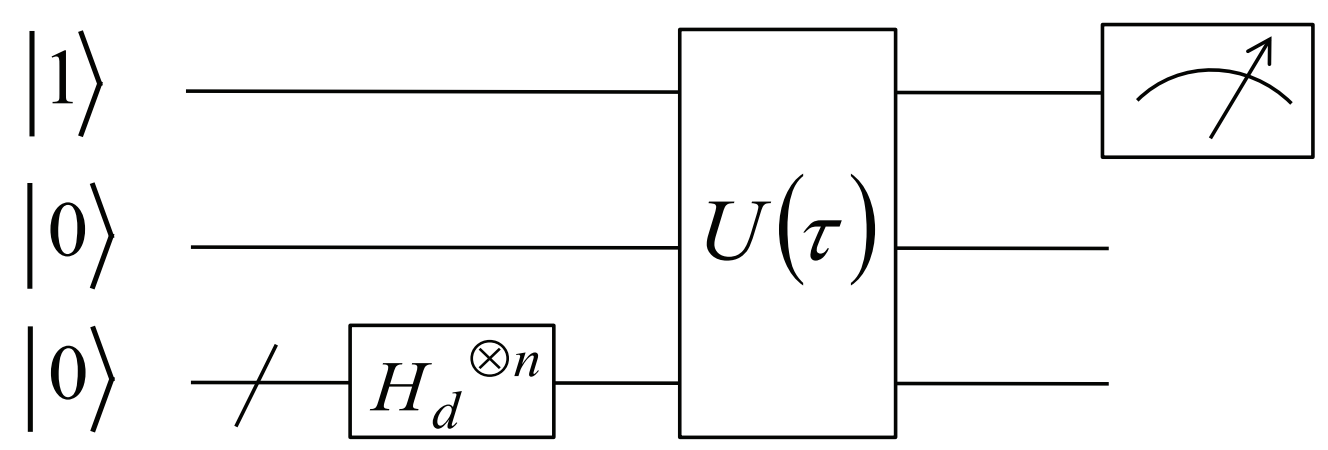

FIG. 1: Quantum circuit for obtaining the energy spectrum of a physical system. The first line represents a probe qubit. $H_{d}$ represents the Hadamard gate, and $U(\tau)$ is a time evolution operator driven by a Hamiltonian given in Eq. (1). The last $n$ qubits represent the system whose spectrum is to be obtained.

where $|j-1\rangle$ are the computational basis. This is achieved by initializing $R$ in state $|0\rangle^{\otimes(n+1)}$ and applying an operator $I_{2} \otimes H_{d}^{\otimes n}$ on $R$, where $H_{d}$ is the Hadamard gate. The states $\left|\varphi_{j}\right\rangle$ are eigenstates of $\widetilde{H}$ with eigenvalues of $\alpha$ and degeneracy of $N$. Therefore, the reference state $\left|\Psi_{0}\right\rangle$ has an eigenvalue $E_{0}=\alpha$.

We then make a guess on the range of the transition frequencies, $\left[\omega_{\min }, \omega_{\max }\right]$, between the reference state $\left|\Psi_{0}\right\rangle$ and the excited states $\left|\Psi_{j}\right\rangle=|1\rangle\left|\lambda_{j}\right\rangle$, of $R$, where $\left|\lambda_{j}\right\rangle(j=1,2, \ldots, N)$ are the $j$-th energy eigenstates of the system with eigenvalues $E_{j}$. As in Ref. [4], this frequency range is discretized into $m$ intervals, where each interval has a width of $\Delta \omega=$ $\left(\omega_{\max }-\omega_{\min }\right) / m$. The center frequencies are $\omega_{k}=\omega_{\min }+(k+1 / 2) \Delta \omega, k=0 \ldots, m-1$, and these frequency points form a frequency set.

We set the frequency of the probe qubit to be $\omega_{k}$, and let the entire system evolve with Hamiltonian $H$ for time $\tau$. Then read out the state of the probe qubit by performing a measurement on the probe qubit in basis of $|0\rangle$ and $|1\rangle$, which represent the ground and excited states of the probe, respectively. We repeat the whole procedure many times to obtain the decay probability of the probe qubit. Then set the probe qubit in another frequency and repeat the above procedure until run over all the frequency points in the frequency set. Once we observe a decay of the probe qubit, it indicates that an excitation between the reference state and an excited state of the register $R$ occurs and the last $n$ qubits of $R$ collapse to an eigenstate of the system.

The procedure of the algorithm is summarized as follows: $(i)$ prepare a probe qubit in 
its excited state $|1\rangle$ and a $(n+1)$-qubit quantum register $R$ in state $|0\rangle^{\otimes(n+1)}$; (ii) apply operator $I_{2} \otimes H_{d}^{\otimes n}$ on the register $R$, then $R$ is prepared in the reference state $\left|\Psi_{0}\right\rangle$; (iii) implement the time evolution operator $U(\tau)=\exp (-i H \tau) ;(i v)$ read out the state of the probe qubit in basis of $|0\rangle$ and $|1\rangle ;(v)$ perform steps $(i)-(i v)$ a number of times to obtain the decay probability of the probe qubit; $(v i)$ repeat steps $(i)-(v)$ for different frequencies of the probe qubit. The quantum circuit for steps $(i)-(i v)$ is shown in Fig. 1.

In this algorithm, we must implement the time evolution operator $U(\tau)=\exp (-i H \tau)$. This operator can be implemented based on the Trotter-Suzuki formula [5]:

$$
U(\tau)=\left[e^{-i \frac{1}{2} \omega \sigma_{z} \tau / L} e^{-i \widetilde{H} \tau / L} e^{-i\left(c \sigma_{x} \otimes A\right) \tau / L}\right]^{L}+O\left(\frac{1}{L}\right)
$$

where $L$ does not depend on the size of the problem. $L$ can be made sufficiently large such that the error is bounded by some threshold [6]. In Eq. (5), the first term of $U(\tau)$, $e^{-i \frac{1}{2} \omega \sigma_{z} \tau / L}$ is diagonal and can be implemented efficiently on a quantum computer. And the second term can be treated as a controlled- $U_{S}$ operation, where $U_{S}=e^{-i H_{S} \tau / L} . H_{S}$ is a Hamiltonian that involves two-body interaction, and can be simulated efficiently on a quantum computer. Therefore the second term of $U(\tau)$ can also be implemented efficiently. For the third term, $e^{-i\left(c \sigma_{x} \otimes A\right) \tau / L}$, the Hamiltonian $c \sigma_{x} \otimes A$ involves many-body interaction. In Ref. [7], it was shown that a many-body interaction Hamiltonian can be simulated efficiently by a Hamiltonian with two-body interactions. The Hamiltonian $c \sigma_{x} \otimes A$ is equal to $c\left(H_{d} \sigma_{z} H_{d}\right) \otimes\left(H_{d} \sigma_{z} H_{d}\right) \otimes\left[H_{d}\left(\begin{array}{cc}\sqrt{2} & 0 \\ 0 & 0\end{array}\right) H_{d}\right]^{\otimes n}$, and the unitary operator $e^{-i\left(c \sigma_{x} \otimes A\right) \tau / L} \operatorname{can}$ be written as $H_{d}^{\otimes(n+2)} \exp \left[-i c \tau \sigma_{z} \otimes \sigma_{z} \otimes\left(\begin{array}{cc}\sqrt{2} & 0 \\ 0 & 0\end{array}\right)^{\otimes n}\right] H_{d}^{\otimes(n+2)}$. It can be implemented using the circuit shown in Fig. 2. In the circuit, the $(n+1)$ and $n$-qubit controlled unitary operators can be implemented efficiently with $O\left(n^{2}\right)$ elementary gates [8]. Therefore the unitary operator $U(\tau)$ can be implemented efficiently on a quantum computer.

\section{EFFICIENCY OF THE ALGORITHM}

As discussed in Ref. [4], the efficiency of the algorithm is defined as the number of times that the circuit in Fig. 1 must be run to obtain the decay probability of the probe qubit, $P_{\text {decay }}$. And it must be at least proportional to $1 / P_{\text {decay }}$. In our algorithm, consider the 


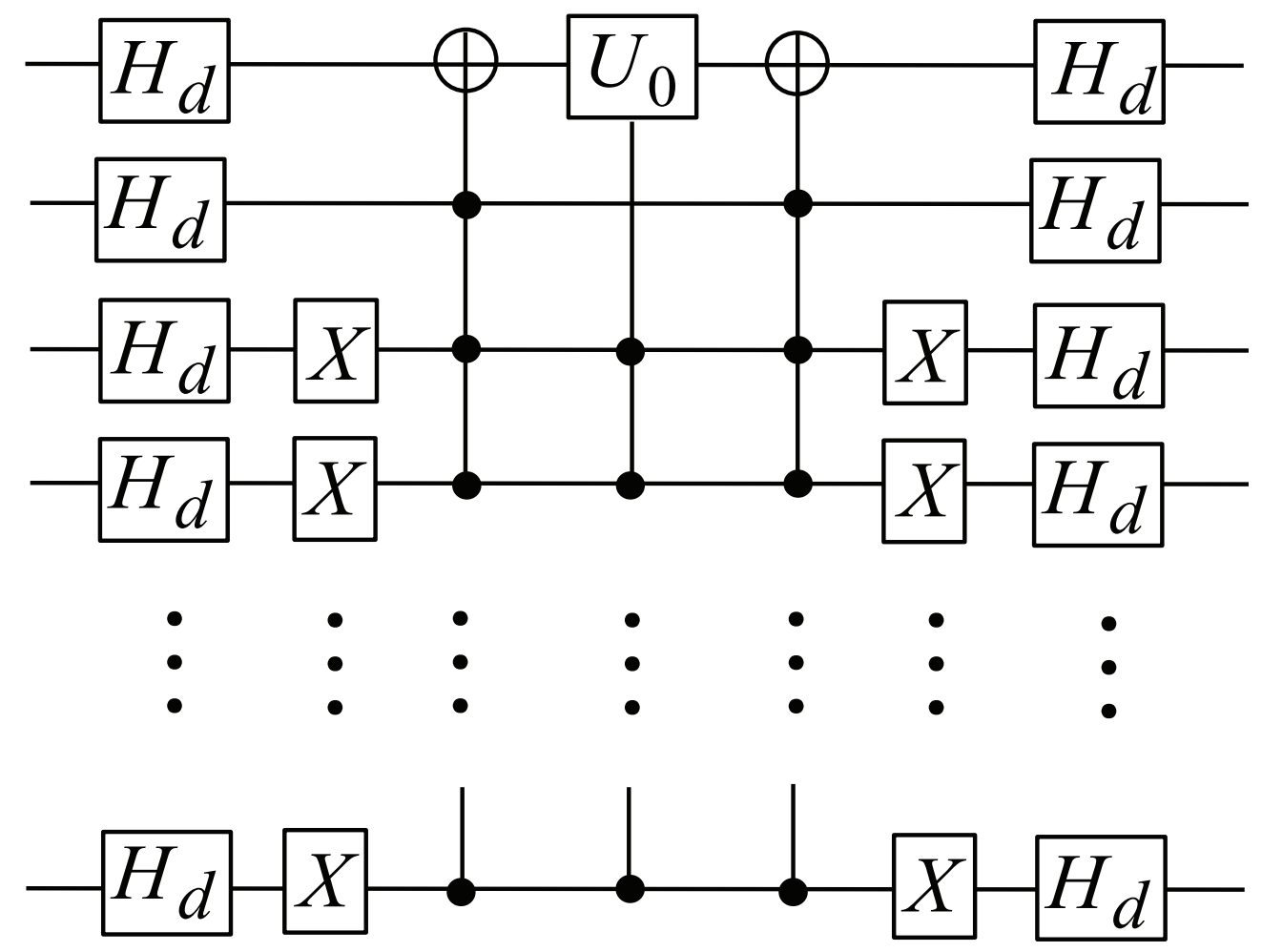

FIG. 2: Quantum circuit for implementing the unitary operator $e^{-i\left(c \sigma_{x} \otimes A\right) \tau / L}$, where operator $A$ is given in Eq. (3), $\sigma_{z}$ is the Pauli matrices and $U_{0}=e^{i \sqrt{2^{n}} c r / L \sigma_{z}}$.

excitation from the reference state $\left|\Psi_{0}\right\rangle$ to the $j$-th excited state of $R,\left|\Psi_{j}\right\rangle$, with the probe qubit frequency being set to $\omega_{k}$, the decay probability of the probe qubit is

$$
P_{\text {decay }}=\sin ^{2}\left(\frac{\Omega_{0 j} \tau}{2}\right) \frac{Q_{0 j}^{2}}{Q_{0 j}^{2}+\left(E_{j}-E_{0}-\omega_{k}\right)^{2}}, j=1,2, \ldots, N
$$

where $Q_{0 j}=2 c\left|\left\langle\Psi_{j}|A| \Psi_{0}\right\rangle\right|$, and $\Omega_{0 j}=\sqrt{Q_{0 j}^{2}+\left(E_{j}-E_{0}-\omega_{k}\right)^{2}}$. Eq. (6) describes the Rabi oscillation process in which the probe qubit exchanges an excitation with the register $R$. The probe decays from its excited state to the ground state, while $R$ is transferred from the reference state $\left|\Psi_{0}\right\rangle$ to the excited state $\left|\Psi_{j}\right\rangle=|1\rangle\left|\lambda_{j}\right\rangle$, and the system collapses to its eigenstate $\left|\lambda_{j}\right\rangle$.

The excited states $\left|\Psi_{j}\right\rangle$ of the register $R$ can be spanned in computational basis as

$$
\left|\Psi_{j}\right\rangle=\sum_{k=1}^{N} d_{j k}\left|\mu_{k}\right\rangle=\sum_{k=1}^{N} d_{j k}|1\rangle|k-1\rangle .
$$


Then $Q_{0 j}$ can be written as

$$
\begin{aligned}
Q_{0 j} & =2 c\left|\left\langle\Psi_{j}|A| \Psi_{0}\right\rangle\right| \\
& =2 c \sum_{k=1}^{N} \sum_{l=1}^{N} \frac{1}{\sqrt{N}} d_{j k} \mid\left\langle 1\left|\left\langle k-1|A| \varphi_{l}\right\rangle\right|\right. \\
& =2 c \sum_{k=1}^{N} d_{j k} .
\end{aligned}
$$

From Eq. (8), we can see that the decay probability and thus the efficiency of the algorithm, depends on the coupling strength $c$, the evolution time $\tau$ and the term $\sum_{k=1}^{N} d_{j k}$, which is the summation of the vector elements of the $j$-th eigenstate of the system. As we have discussed in Ref. [4], as long as the number of "energy levels of interest" is polynomially large, the complexity of the algorithm grows polynomially with the size of the system.

It should be pointed out that the coupling strength $c$ is small $(c \ll \omega)$ and the effect of the perturbation of the probe qubit to the register $R$ is sufficiently weak. In this case, its effect on $R$ can be calculated to a first approximation, by ignoring all the other energy levels of the register $R$.

The coupling between the reference state and all the other energy levels except the one that resonant with the probe, contributes to the decay probability of the probe qubit, therefore introduces an error, $P_{\text {decay }}^{\text {err }}$, in $P_{\text {decay }}$. For a system with discrete energy levels, when there is no energy level that has exponentially large degeneracy, the error $P_{\text {decay }}^{\text {err }}$ can be constrained to be very small because $c$ can be set polynomially small. It was shown that the simulation of any Hamiltonian may be performed linearly in the scaled time $\tau$ [9]. And in our algorithm we have $c \tau \sim 1$. Therefore, in this case, the algorithm can be run in finite time $\tau$. For a system with exponentially large number of degenerated states, we may not find a coupling coefficient $c$ that is polynomially small such that the evolution time is finite [10].

We consider the case where the transition between states $\left|\Psi_{0}\right\rangle$ and $\left|\Psi_{1}\right\rangle$ resonates with the probe qubit with frequency of $\omega$, such that $E_{1}-E_{0}=\omega$. The error $P_{\text {decay }}^{\text {err }}$ can be calculated 
as follows

$$
\begin{aligned}
P_{\text {decay }}^{\mathrm{err}} & =\sum_{j=2}^{N} \sin ^{2}\left(\frac{\Omega_{0 j} \tau}{2}\right) \frac{Q_{0 j}^{2}}{Q_{0 j}^{2}+\left(E_{j}-E_{0}-\omega\right)^{2}} \\
& <\sum_{j=2}^{N} \frac{Q_{0 j}^{2}}{\left(E_{j}-E_{1}\right)^{2}} \\
& =4 c^{2} \sum_{j=2}^{N} \frac{\left(\sum_{k=1}^{N} d_{j k}\right)^{2}}{\left(E_{j}-E_{1}\right)^{2}} .
\end{aligned}
$$

For a finite system, if the ground state is not degenerate and the term $\sum_{j=2}^{N} \frac{\left(\sum_{k=1}^{N} d_{j k}\right)^{2}}{\left(E_{j}-E_{1}\right)^{2}}$ is bounded by a finite number $M$. The term $4 c^{2} M$ can be small by setting $c$ small. In this case, the algorithm can be completed in finite time. That is, for a finite system, a sufficiently small $c$ and a finite evolution time $\tau$ exist.

\section{EXAMPLE: OBTAINING THE ENERGY SPECTRUM OF THE WATER MOLECULE}

In the following, using the water molecule as an example, we simulate the algorithm for obtaining the energy spectrum of a system that we have no information about its eigenstates.

The Hamiltonian of the water molecule is the same as shown in Ref [4]. Considering the $\mathrm{C}_{2 V}$ and ${ }^{1} A_{1}$ symmetries, the Hartree-Fock wave function for the ground state of the water molecule is $\left(1 a_{1}\right)^{2}\left(2 a_{1}\right)^{2}\left(1 b_{2}\right)^{2}\left(3 a_{1}\right)^{2}\left(1 b_{1}\right)^{2}$. Using the STO-3G basis set [11] and freezing the first two $a_{1}$ orbitals, a model space with ${ }^{1} A_{1}$ symmetry that includes the $3 a_{1}, 4 a_{1}, 1 b_{1}$ and $1 b_{2}$ orbitals is constructed by considering only single and double excitations to the external space. For simplicity, we remove two of the highest excitations, then the dimension of the state space of the water molecule is 16 . Therefore four qubits are required to simulate the water molecule in this calculation.

We set the reference energy $E_{0}=\alpha=-100$, and vary the frequency of the probe qubit in the range $\omega \in[15.8,19.2]$, which is divided into 170 equal intervals. The coupling strength and the evolution time are set as $c=0.002$ and $\tau=1200$ (here energies and time are measured in units of Hartree and Hartree ${ }^{-1}$, respectively). Then we run the algorithm and obtain the spectrum of the transition frequencies between the reference state and the eigenstates of the water molecule. The results are shown in figure 1. From the figure we 


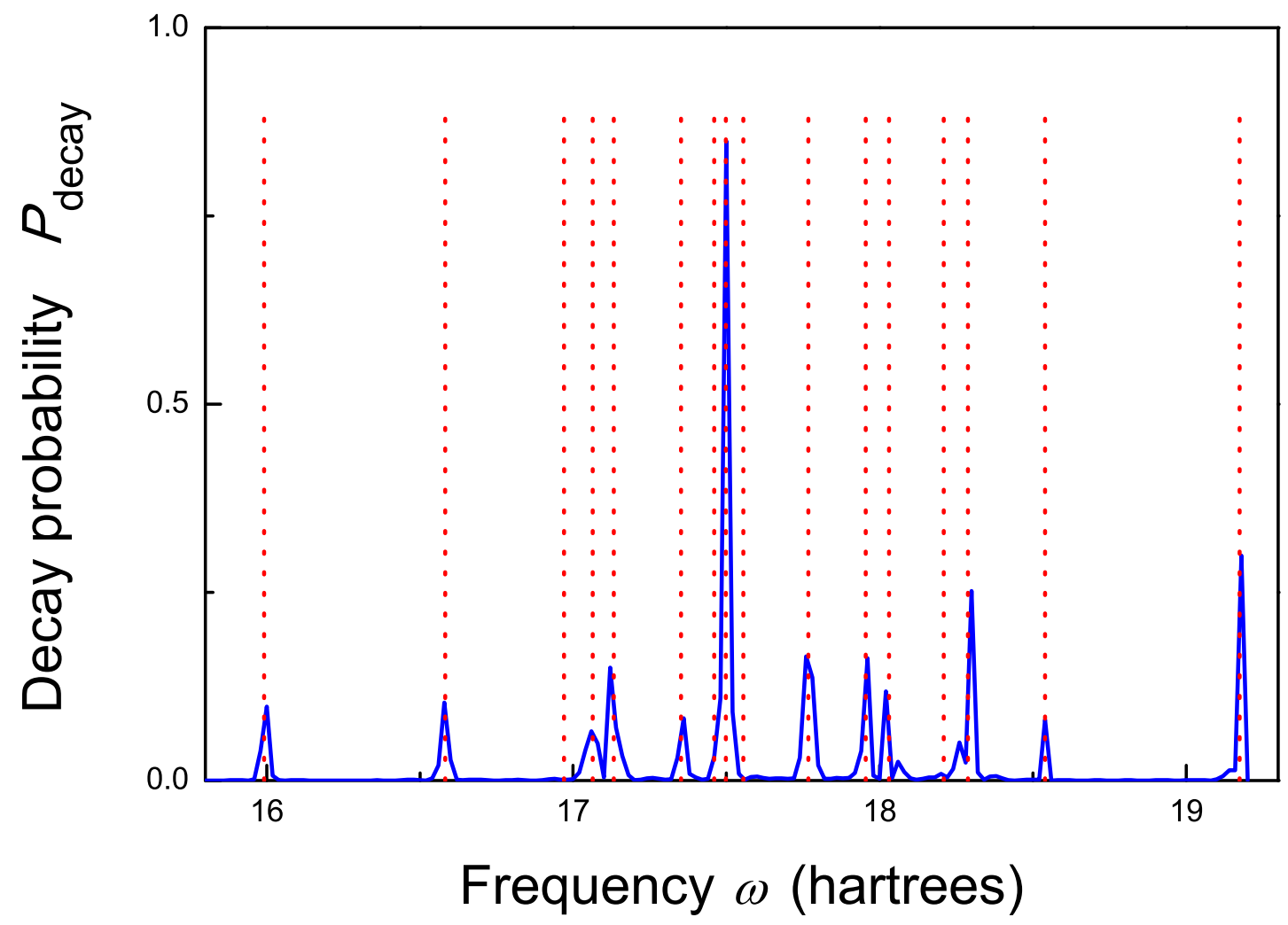

FIG. 3: (Color online) Transition frequency spectrum between the reference state, $\left|\Psi_{0}\right\rangle$, and the 16 eigenstates of the water molecule. The blue solid curve represents the decay probability of the probe qubit at different frequencies with the coupling coefficient $c=0.002$ and the evolution time $\tau=1200$. The frequency for the probe qubit is set in the range $\omega \in[15.8,19.2]$, and is divided into 170 equal intervals. The red dotted vertical lines represent the known transition frequencies between the reference state and all the 16 eigenstates of the water molecule.

can see that most of the spectrum obtained using our algorithm are in good agreement with the known transition frequency spectrum (in red) of the water molecule, except that four peaks (the 3rd, 7-th, 9-th, and 13-th) are missing.

Some missing peaks can be found by only increasing the density of the frequency points in certain frequency range. We vary the frequency of the probe qubit in the range $\omega \in$ $[15.8,17.0]$ and divide this frequency range into 240 equal intervals, run the algorithm. The results are shown in figure $2(a)$. We can see that the 3 rd peak at $\omega=16.9705$ is visible now. We then vary the frequency of the probe in the range $\omega \in[17.2,18.0]$, which is divided into 160 equal intervals, run the algorithm, from the results shown in figure 2(b), we can see that the 9 -th peak at $\omega=17.5552$ appears. We vary the frequency of the probe in 

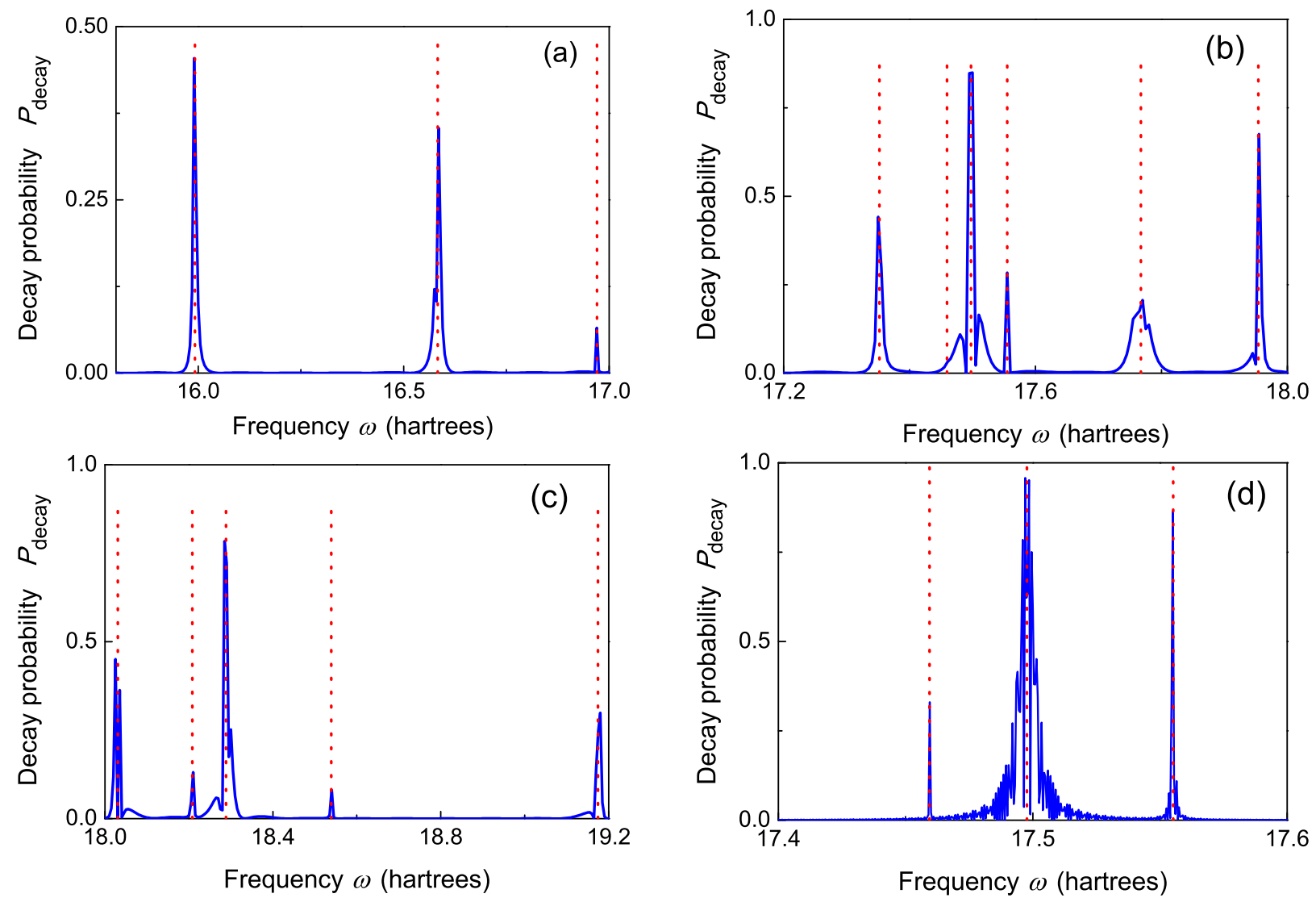

FIG. 4: (Color online) Same as in Fig. 3, except that in $(a)$ and $(b), c=0.002$ and $\tau=1200$. In (a) the frequency of the probe qubit $\omega$ is set in the range $\omega \in[15.8,17.0]$ and is divided into 240 equal intervals; in $(b) \omega \in[17.2,18.0]$ and is divided into 160 equal intervals. In $(c), c=0.001$, $\tau=2400, \omega \in[18.0,19.2]$ and is divided into 240 equal intervals. In $(d), c=0.001, \tau=20000$, $\omega \in[17.4,17.6]$ and is divided into 400 equal intervals.

the range $\omega \in[18.0,19.2]$, which is also divided into 240 equal intervals, and set $c=0.001$ and $\tau=2400$. From the results shown in figure $2(c)$, we can see that the 13 -th peak at $\omega=18.2082$ is clearly visible now.

In the case where the term $\sum_{k=1}^{N} d_{j k}$ is small (then the decay probability is small), increasing the evolution time $\tau$ can increase the height of the peak. For the 7 -th eigenstate of the system, we have $\sum_{k=1}^{N} d_{j k}=0.0305153$, which is a small number. We vary the frequency of the probe in the range $\omega \in[17.4,17.6]$, which is divided into 400 equal intervals, set $c=0.001$ and $\tau=20000$, then run the algorithm. The results are shown in figure $2(d)$. We can see that the 7 -th peak at $\omega=17.4594$ appears now. 


\section{DISCUSSION}

As we have demonstrated in the example for obtaining the energy spectrum of water molecule in the supplementary material, by varying the coupling strength $c$, the evolution time $\tau$ and increasing the density of the frequency points, one can obtain the entire energy spectrum and the corresponding eigenstates of a system. As we have discussed in Ref. [4], the accuracy of the algorithm is defined by the parameters $c$ and $\tau$. We need to set $c$ to be small so that the system-probe coupling is weak, and the evolution time $\tau$ to be large. The size of the frequency intervals $\Delta \omega$ is set by the choice of $c$ and $\tau: \Delta \omega$ should be smaller than the width of the peaks in order to avoid missing some of the peaks.

It should be pointed out that our algorithm cannot be used for obtaining the eigenenergies of eigenstates that have anti-symmetric symmetry. As shown in Eq. (8), the term $Q_{0 j}$ is zero therefore the decay probability of the probe is zero. By using some other techniques, such as group theory, one can determine the anti-symmetric state and using the PEA to obtain its eigenenergy. Also, in our algorithm, one cannot tell whether a given energy level is degenerate or not. It is also difficult to separate the near-degenerate states. In these cases, by applying our algorithm, once the probe qubit collapses to its ground state, the system is in a superposition of the degenerated eigenstates of the system. One can use this state as the input for the PEA to resolve the eigenenergy and the corresponding eigenstates.

We now compare this algorithm with the algorithm we proposed in Ref. [4]. In the previous algorithm, we prepare the system in an initial state that is close to one of its eigenstates, and construct an excitation operator that transfers the initial state to another state of the system. By coupling with a probe qubit, the system is evolved to the desired eigenstates of the system. The form of the excitation operator depends on the guess state and the part of the energy spectrum that is of interest.

In this algorithm, one ancilla qubit is added to the register of the system to construct a quantum register $R$, and $R$ is coupled to a probe qubit. Here $R$ can be considered as an "artificial system" which plays the same role as "the system" in the previous work [4].

The Hamiltonian of the register $R$ given in Eq. (2) can be written as: $\widetilde{H}=\alpha|0\rangle\langle 0| \otimes I_{N}+$ $\sum_{j=1}^{N} E_{j}|1\rangle\left\langle 1|\otimes| \lambda_{j}\right\rangle\left\langle\lambda_{j}|=\alpha| 0\right\rangle\left\langle 0\left|\otimes I_{N}+\sum_{j=1}^{N} E_{j}\right| \Psi_{j}\right\rangle\left\langle\Psi_{j}\right|\left(E_{j}\right.$ are the eigenenergies of the system). Its ground state is $N$-fold degenerate and in the form of $|0\rangle|j-1\rangle(j=1,2, \ldots, N)$, which are the eigenstates of the first term of $\widetilde{H}$. And its excited states are $\left|\Psi_{j}\right\rangle=|1\rangle\left|\lambda_{j}\right\rangle\left(\left|\lambda_{j}\right\rangle\right.$ 
are the eigenstates of the physical system), which are the eigenstates of the second term of $\widetilde{H}$. The register $R$ is prepared in the reference state $\left|\Psi_{0}\right\rangle$ which is the eigenstate of the first term with eigenvalue $\alpha$. We introduced an excitation operator $A$ as defined in Eq. (3) acting on the register $R$. From the expansion of $A$, one can see that $A$ contains $N$ terms which provide all possible excitations between the subspace of $|0\rangle\langle 0| \otimes I_{N}$ and the subspace of $|1\rangle\langle 1| \otimes H_{S}$. The probe qubit is coupled to $R$ with interaction operator $c \sigma_{x} \otimes A$, which transfers the probe from the excited state to its ground state and $R$ from the reference state to a state in the subspace of $|1\rangle\langle 1| \otimes H_{S}$. The overlap of this state with the excited state $\left|\Psi_{j}\right\rangle$ is $\left\langle\Psi_{j}|A| \Psi_{0}\right\rangle$ and has been derived in Eq. (8). By employing the operator $A, R$ can be evolved to any of its excited states starting from the initial state $\left|\Psi_{0}\right\rangle$. The probe qubit exhibits a dynamical response only when it is resonant with a transition between the reference state $\left|\Psi_{0}\right\rangle$ and a state $\left|\Psi_{j}\right\rangle$ of $R$. Therefore, when $E_{j}-\alpha=\omega$ ( $\omega$ is the frequency of the probe qubit), the probe qubit decays to its ground state with decay probability $P_{\text {decay }}=\sin ^{2}\left(\frac{Q_{0 j} \tau}{2}\right)$ while the register $R$ is transferred to state $\left|\Psi_{j}\right\rangle$. Therefore for a finite system, as long as the term $Q_{0 j}$ is not exponentially small, the algorithm can be run efficiently. Another advantage of employing operator $A$ is that in the Trotter expansion, the unitary operator related to the interaction operator can be implemented efficiently as we have shown in Fig. (2).

In this algorithm, we introduced a reference state $\left|\Psi_{0}\right\rangle$ and an excitation operator $A$, both do not depend on systems. They are general and can be applied for any arbitrary physical system. This algorithm provides a general approach for obtaining the energy spectrum and energy eigenstates of a physical system without having any information about the eigenstates of the system.

\section{Acknowledgments}

We thank Sahel Ashhab and L.-A. Wu for helpful discussions. This work was supported by "the Fundamental Research Funds for the Central Universities" of China and the National Nature Science Foundation of China (Grants No. 11275145 and No. 11305120).

[1] E. R. Davidson, J. Comp. Phys. 17, 87 (1975).

[2] D. S. Abrams and S. Lloyd, Phys. Rev. Lett. 83, 5162 (1999). 
[3] H. Wang, S. Kais, A. Aspuru-Guzik, and M. R. Hoffmann, Phys. Chem. Chem. Phys. 10, 5388 (2008).

[4] H. Wang, S. Ashhab, and F. Nori, Phys. Rev. A 85, 062304 (2012).

[5] M. Nielsen, I. Chuang, Quantum Computation and Quantum Information (Cambridge Univ. Press, Cambridge 2000).

[6] S. Lloyd, Science 273, 1073-1078 (1996).

[7] S. Bravyi, D. P. DiVincenzo, D. Loss, and B. M. Terhal, Phys. Rev. Lett. 101, 070503 (2008).

[8] A. Barenco, et al., Phys. Rev. A 52, 3457 (1995).

[9] D. W. Berry, G. Ahokas, R. Cleve, and B. C. Sanders, Comm. in Math. Phys. 270, 359 (2007)

[10] H. Wang, submitted.

[11] A. Szabo and N. Ostlund, Modern Quantum Chemistry: Introduction to advanced Electronic Structure Theory (McGraw-Hill, New York, 1989). 